\title{
Corticoides intratimpánicos: una revisión sistemática
}

\author{
Intratympanic Corticosteroids: A Systematic Review
}

Rubén González', Jorge Caro²

\begin{abstract}
RESUMEN
Muchas patologías otológicas como la hipoacusia súbita y la enfermedad de Ménière han sido tratadas con corticoides sistémicos, los que tienen diversos efectos secundarios potencialmente graves. Los corticoides intratimpánicos son una alternativa en el tratamiento para estas enfermedades del oído interno, con lo que se podría aumentar su efecto al mejorar el perfil farmacocinético de la droga disminuyendo los efectos adversos sistémicos. Para evaluar la evidencia disponible con respecto a la utilidad y racionalidad del uso de esta terapia se realizó una revisión sistemática respecto al tema. Hay pocos trabajos de buena calidad metodológica, los que muestran una efectividad similar de los corticoides intratimpánicos comparados con los sistémicos en terapia primaria y una superioridad ante placebo en terapia de rescate. Por otra parte hay gran variación en los protocolos de administración de la droga y el tipo de corticoide usado. Considerando la alta tasa de resolución espontánea de las patologías en que se ha probado su uso y los resultados no siempre concordantes de los estudios es difícil establecer la real utilidad de esta terapia. Se requieren estudios de mayor calidad metodológica que ocupen protocolos estandarizados de administración de corticoides intratimpánicos para definir la mejor aplicación de esta modalidad de terapia.
\end{abstract}

Palabras Clave: Hipoacusia súbita, corticoides intratimpánicos, tratamiento corticoides intratimpánicos, enfermedad de Ménière.

\begin{abstract}
Many otologic diseases as sudden sensorineural hearing loss and Ménière disease had been treated with systemic corticosteroids, which have many potentially serious adverse effects. The intratympanic corticosteroids are an alternative for the treatment of these diseases of the internal ear, having less systemic adverse events and better pharmacokinetic profile, which may promote its therapeutic effects. We made a systematic review searching the existing evidence about the utility and rationality of the clinical use of this therapy. The few good quality existing trials show a similar effectiveness of the intratympanic corticosteroids compared with the systemic ones for primary therapy and a superiority compared with placebo for rescue therapy. There is a great variation between the different protocols of administration of the intratympanic
\end{abstract}

1 Interno de Medicina. Pontificia Universidad Católica de Chile

2 Médico del Departamento de Otorrinolaringología de la Pontificia Universidad Católica de Chile 
corticosteroids and the type of corticosteroid used. Considering the high spontaneous resolution of the symptoms in this pathologies and the disparity between the results in the different trials its difficult to establish the real utility of this therapy. More good quality studies with standardized protocols are needed to determine the best clinical application for this kind of therapy.

Key words: sudden sensoryneural hearing loss, intratympanic coticosteroids, Ménière desease

\section{INTRODUCCIÓN}

En 1991, Itoh ${ }^{1}$ describió el uso de corticoides intratimpánicos para tratar pacientes con enfermedad de Ménière (EM). Posteriormente se reportaron usos en otras patologías como hipoacusia súbita (HS), tinnitus, etc. ${ }^{2} y$ desde entonces han aparecido diversos trabajos que intentan evaluar el uso de esta modalidad de terapia en dichas patologías, inicialmente a través de reporte de casos, estudios retrospectivos y prospectivos hasta llegar a la realización de estudios clínicos randomizados para algunas de ellas.

En muchos casos la evidencia muestra resultados no totalmente convincentes, por lo que su uso ha sido bastante controversial hasta el día de hoy, no existiendo un acuerdo universal respecto a su utilidad ni protocolo de administración, lo que es comprensible considerando lo novedoso de la terapia.

Por esto hemos querido realizar una revisión sistemática narrativa del tema, buscando los trabajos disponibles en las bases de datos Pubmed y Cochrane Library y en las referencias de los trabajos encontrados, seleccionando aquellos de mayor calidad metodológica de acuerdo a los criterios de la medicina basada en evidencias y analizando sus resultados, además de incluir aquellos trabajos preclínicos y que hablan de aspectos farmacológicos de los corticoides intratimpánicos con el objetivo de comprender la lógica que fundamenta su uso, los beneficios y riesgos asociados, y así tener mayores elementos de juicio para definir cuándo utilizarlos en nuestros pacientes.

\section{METODOLOGÍA}

Se realizó una exhaustiva búsqueda en Pubmed y la Cochrane Library utilizando 30 nomenclaturas farmacológicas distintas de corticoides cruzado con los términos intratimpánico, transtimpánico y/ 0 oído medio. Además se realizó una búsqueda en las referencias de los trabajos encontrados para aumentar la sensibilidad de la búsqueda y se consultó a expertos en el tema.

Posteriormente se seleccionaron tanto los trabajos clínicos de mayor calidad metodológica que evalúan los usos de los corticoides intratimpánicos en la práctica clínica, así como también aquellos trabajos que investigan respecto a los aspectos farmacológicos de los mismos, cuya síntesis y análisis se entrega en la presente revisión.

\section{FARMACOLOGÍA Y ESTUDIOS PRECLÍNICOS}

Los mecanismos mediante los cuales los corticoides ejercen sus efectos en el oído medio han sido estudiados en animales y en humanos. Entre los mecanismos se ha descrito la activación y aumento en la expresión del intercambiador sodio/ hidrógeno (NHE-3) en células epiteliales humanas de oído medio en forma dosis dependiente, alterando así el transporte periciliar de fluidos ${ }^{3}$. Además en cultivos de células de oído medio que permiten estudiar el transporte vectorial de electrolitos (líneas celulares MESV) se ha visto que los corticoides aumentan la expresión de la subunidad alfa de los canales de sodio, aumentando así el transporte transepitelial de este ion ${ }^{4}$. Estos efectos podrían facilitar la reabsorción de fluidos del oído medio, contribuyendo a la prevención de Otitis Media con Efusión (OME). Park SN y col demostró en ratas que sufren de otitis media neumocócica que la administración de antibióticos sistémicos solos y combinados con corticoides sistémicos genera menores cambios histológicos inflamatorios y de metaplasia del epitelio del oído medio a epitelio secretor al compararlos con ratas que no reciben dichos tratamientos. Estos cambios 
son mucho menores al administrarse la combinación con corticoides, especialmente en lo que se refiere a la metaplasia a epitelio secretor, lo que generaría una disminución del tiempo que permanece la efusión en el oído medio posterior a una Otitis Media Aguda (OMA) $)^{5}$.

En chinchilla se ha demostrado que los corticoides locales disminuyen la producción de glicoproteínas que forman la secreción mucosa del oído medio mediante un mecanismo dependiente de la inhibición de fosfolipasa $A 2^{6}$. Esto llevó a otros investigadores a evaluar los niveles de leucotrieno B4 (LTB4), producido a partir del ácido araquidónico a través de la fosfolipasa $A 2$, en niños que sufrían OMA, encontrando que los niveles de LTB4 no son afectados por los corticoides, pero sí disminuyen el tiempo de duración de OME post-OMA ${ }^{7}$, por lo que tal vez este efecto sea por los mecanismos mencionados en el párrafo anterior, no dependientes de LTB4.

Otros efectos descritos son el aumento en $29,26 \%$ del flujo sanguíneo coclear y que persiste por lo menos por una hora en cerdos de guinea, sin generar cambios histológicos ni auditivos ${ }^{8}$; la disminución de la génesis de colesteatoma experimental al ser utilizado localmente luego de la aplicación de irritantes como el propilen glicol ${ }^{9}$; y el aumento de la expresión de aquaporina 1 en la cóclea de la rata en forma dosis dependiente, lo que podría explicar parte de sus efectos en la EM al alterar la homeostasis del agua del oído interno ${ }^{10}$.

Al analizar los aspectos farmacocinéticos, vemos que luego de la aplicación de corticoides en el oído medio su concentración en perilinfa sigue un modelo de distribución de un compartimento, es decir, traspasa hacia el oído interno sin difundir a otros compartimentos corporales ${ }^{11}$. Éste transporte hacia el oído medio estaría mediado por la ventana redonda, compuesta por tres capas: epitelio externo, epitelio interno y tejido conectivo que las separa. El paso de sustancias por esta membrana estaría influenciado por el tamaño, concentración y carga eléctrica de la sustancia, grosor de la membrana, y el uso de agentes facilitantes ${ }^{12}$. Estudios en cerdo de guinea muestran que el mejor perfil farmacocinético lo tiene la metilprednisolona al compararla con la dexametasona y la hidrocortisona ${ }^{13}$. Respecto a la vía de administración, vemos que la concentración perilinfática es mucho mayor luego de la aplicación transtimpánica, alcanzando un área bajo la curva 593 veces más alta que luego de la administración endovenosa. Además, si se utiliza dexametasona disuelta en gel termosensible el tiempo medio de eliminación aumenta 28,6 veces ${ }^{14}$. Otras formas de incrementar la concentración perilinfática de corticoides es utilizando histamina ${ }^{15} 0$ papaverina tamponada con bicarbonato ${ }^{16}$.

\section{USOS CLÍNICOS}

El uso de corticoides intratimpánicos aparece como una alternativa interesante para el tratamiento de diversas patologías otológicas que requieren corticoides sistémicos, ya que evitaría los efectos secundarios de estos (hemorragia digestiva, necrosis avascular de cadera, alza de glucosa, cambios en el estado de ánimo, insomnio, etc.), lo cual sería especialmente útil en aquellos pacientes más lábiles (por ej: diabéticos) y alcanzaría más altas concentraciones locales pudiendo así aumentar sus efectos. Además es un procedimiento que requiere sólo anestesia local, se realiza ambulatoriamente en una consulta y es relativamente fácil de realizar.

Sin embargo, su uso tiene riesgos que no tienen los corticoides sistémicos, como por ejemplo la perforación timpánica persistente, disgeusia, dolor y vértigo, siendo estos dos últimos de corta duración por lo general. Algunos de estos riesgos son serios, pero tienen una baja incidencia. Las perforaciones timpánicas persistentes no siempre son reportadas, y dentro que los trabajos que las reportan como complicación, estas fluctúan entre $0 \%$ y $36 \%$, siendo este porcentaje mayor en pacientes con disfunción tubaria crónica ${ }^{17-19}$. Por otra parte, varios expertos consultados señalan más de un caso con esta complicación, los que no han sido publicados.

Por otra parte, vemos que las principales patologías en que se ha intentado usar los corticoides intratimpánicos (EM e HS) tienen una fisiopatología aún no del todo dilucidada, y que aparentemente pueden ser verdaderos síndromes generados por distintas causas por lo que es difícil idear un tratamiento dirigido, específico para cada uno de ellos. Así se explica que el uso de corticoides tanto 
sistémicos como locales en estas patologías tenga resultados, en ocasiones, discordantes.

Además vemos que no hay un protocolo universalmente aceptado para la aplicación de los corticoides intratimpánicos, variando en los diversos estudios el tipo de corticoide usado (metilprednisolona o dexametasona), la concentración de las soluciones (2 a $24 \mathrm{mg} / \mathrm{ml}$ de dexametasona y 32 a $62,5 \mathrm{mg} / \mathrm{ml}$ de metilprednisolona), volumen total administrado por sesión, que en todo caso no debe superar el volumen total aproximado de la caja timpánica $(0,3$ a $0,5 \mathrm{ml})$ número de sesiones e intervalo entre cada sesión, técnica de entrega de la droga (inyección transtimpánica, colleras, microcatéter a la ventana redonda, infusión contínua). Todo esto también puede contribuir a la heterogeneidad en los resultados de los distintos trabajos.

A continuación revisaremos los trabajos clínicos que han evaluado su uso en cada una de estas enfermedades por separado.

\section{HIPOACUSIA SÚBITA}

La HS es una enfermedad cuya causa exacta es aún desconocida, postulándose etiologías virales, vasculares, inmunes, etc. Su incidencia en Estados Unidos de 5-20 por cada 100.000 al año, siendo desconocidas las cifras locales.

Esta enfermedad se define clínicamente como una baja de 300 más decibeles $(\mathrm{dB})$ en 30 más frecuencias y que se desarrolla en un máximo de 72 horas. Sin embargo algunos trabajos utilizan definiciones distintas, tomando en cuenta las bajas de 200 más $\mathrm{dB}$ y algunos se conforman con que sea en 1 solo tono, lo que atrae mayor heterogeneidad a los trabajos al variar los pacientes incluidos. La definición de mejoría también varía, siendo considerado un éxito en algunos trabajos el alza de cualquier magnitud en el PTP o en la discriminación, mientras que otros son más exigentes y sólo aceptan una mejoría del $50 \%$ o más de audición respecto al oído contralateral en los casos en que este sea sano, 0 bien de cierta cantidad de decibeles que varía de $10 \mathrm{~dB}$ a $30 \mathrm{~dB}$ o de $10 \%$ a $20 \%$ de discriminación. Otro factor que suma heterogeneidad a los trabajos es la diferencia entre las soluciones inyectadas, habiendo sido probado incluso el uso de soluciones de corticoides con ácido hialurónico ${ }^{20}$.

Esta enfermedad tiene una alta tasa de mejoría espontánea que puede incluso estar subestimada, ya que muchos pacientes pueden tener molestias que ceden espontáneamente antes de consultar. En los distintos trabajos se muestran tasas de entre $30 \%$ a $60 \%{ }^{21}$ de recuperación espontánea, siendo menor en aquellos pacientes con hipoacusia severa o profunda (10\% para estos últimos), de mayor edad, con otros síntomas asociados (ej: vértigo), y que reciben terapia tardíamente. Si no responden a la terapia inicial de corticoides sistémicos en un lapso de 10 días, las tasas de mejoría espontánea caen a $0^{19}$ a $6,1 \%{ }^{22}$.

Se ha aceptado que los corticoides sistémicos (orales 0 endovenosos) y los antivirales llevan a un aumento de las tasas de recuperación de esta enfermedad, sin embargo una revisión de la Cochrane ${ }^{23}$ mostró que de toda la literatura disponible sólo hay 2 trabajos de buena calidad metodológica que evalúan el uso de corticoides sistémicos en hipoacusia súbita. Uno de ellos muestra mayor porcentaje de mejoría (61\% versus $32 \%$ ) mientras que el otro no muestra utilidad de los corticoides, por lo que se concluye que no existe evidencia convincente respecto a la utilidad de estos fármacos para esta desafiante enfermedad. Además vemos que la tasa de recuperación en el grupo tratamiento del trabajo que mostró beneficio cae dentro de las tasas de recuperación espontáneas descrita por trabajos previos. Así, vemos que incluso esta modalidad de tratamiento tan universalmente aceptada tiene sus reconvenciones.

Para aquellos pacientes que no responden a la terapia inicial, o bien que tienen contraindicaciones al uso de corticoides, surge la alternativa de los corticoides intratimpánicos.

Kakehata S, y col ${ }^{24}$ comparó la efectividad del uso de dexametasona endovenosa versus intratimpánica en pacientes diabéticos con HS. La dexametasona intratimpánica (4 mg/ml) era administrada mediante inyección por miringotomía asistida por láser 0 bien a través de un tubo de ventilación por 8 días seguidos. En el caso de la dexametasona endovenosa, se dieron $8 \mathrm{mg} /$ día seguido de dosis descendientes por 10 días. Los resultados mostraron que de los 10 pacientes que recibieron 
dexametasona intratimpánica, los $10(100 \%)$ mejoraron en $10 \mathrm{~dB}$ o más de audición (con un promedio de $40 \pm 4,9 \mathrm{~dB}$ ) versus un $67 \%$ (14 pacientes) de los 21 que recibieron tratamiento endovenoso, con una ganancia total promedio de $25,1 \pm 4,8 \mathrm{~dB}(\mathrm{p}<0,05)$. Además $40 \%$ del grupo que recibió terapia local y $29 \%$ del grupo de terapia sistémica mejoró completamente 0 al menos $30 \mathrm{~dB}$ del basal, siendo esta diferencia no estadísticamente significativa, pero que puede deberse al bajo poder del trabajo por el tamaño muestral reducido.

Fuera de este, la mayoría de los trabajos evalúa el uso de corticoides intratimpánicos en pacientes en que ha fallado la terapia sistémica, siendo muchos estudios retrospectivos sin grupo control. Entre ellos destacan 4 estudios prospectivos con grupo contro| $\left.\right|^{21,25-27}$, uno de ellos recientemente publicado (Kiliç $R$ y cols, 2007), que se realizaron en pacientes en que falló la terapia inicial con corticoides sistémicos, mostrando porcentajes de mejoría con el uso de corticoides intratimpánicos que varían entre $39,4 \%$ a $73,6 \%$ mientras que en el grupo control la tasa de mejoría es de 0 a $6,1 \%$, siendo estadísticamente significativo en todos los casos. Además el promedio de mejoría en decibeles también fue mayor en el grupo tratado con corticoides locales (ver Tabla 1). Por otra parte, se ve que el beneficio obtenido es estable al menos a los tres a 24 meses de seguimiento luego de la terapia intratimpánica.

Vemos así que los pocos trabajos con mejor calidad metodológica muestran un efecto positivo de los corticoides intratimpánicos en HS tanto en terapia primaria como de rescate.

\section{ENFERMEDAD DE MÉNIÈRE}

Al igual que la HS, la EM no tiene causa conocida. Sin embargo existe consenso en que sea cual sea su causa existiría una distensión o ruptura del espacio endolinfático secundario a una alteración de la homeostasis de los fluidos del oído interno.

Se han publicado diversos estudios sugiriendo una causa inmune de esta enfermedad, observándose que en pacientes con EM existen autoanticuerpos circulantes contra proteínas del oído interno, habiéndose logrado generar hidrops endolinfático en animales mediante la inducción de autoinmunidad contra proteínas del oído interno ${ }^{28}$. Además se han descrito receptores de corticoides en el oído interno, hay evidencia de cambios histológicos en la estría vascular secundario al uso de corticoides y una creciente experiencia clínica en el uso de corticoides sistémicos para tratar la $E^{29}{ }^{29}$. Tomando en cuenta todo esto, es factible considerar el uso de los corticoides en esta enfermedad, pudiendo mejorarse su farmacocinética mediante la administración local del fármaco.

Las manifestaciones clínicas de la enfermedad se caracterizan por una hipoacusia fluctuante, vértigo intermitente y tinnitus. Las fluctuaciones características en la sintomatología de esta enfermedad llevan a que sea más difícil aún evaluar la efectividad de las distintas terapias, especialmente a largo plazo.

Tabla 1. Estudios prospectivos que evalúan el uso de corticoides intratimpánicos para Hipoacusia Súbita

\begin{tabular}{|c|c|c|c|c|c|c|}
\hline Estudio & $\begin{array}{l}\text { Tasa Mejoría }{ }^{1} \\
\text { Grupo } \text { CIT }^{2}\end{array}$ & $\begin{array}{l}\text { Tasa Mejoría } \\
\text { Grupo Control }\end{array}$ & Valor $p$ & $\begin{array}{l}\text { Decibeles promedio } \\
\text { de Mejoría Grupo CIT }\end{array}$ & $\begin{array}{l}\text { Decibeles promedio de } \\
\text { Mejoría Grupo Control }\end{array}$ & Valor $p$ \\
\hline $\begin{array}{l}\text { Ho HG, y col } \\
2001\end{array}$ & $\begin{array}{l}53,3 \% \\
(8 \text { de } 15)\end{array}$ & $\begin{array}{l}7,1 \% \\
(1 \mathrm{de} 14)\end{array}$ & $<0,05$ & $28,4 \mathrm{~dB}$ & $13,2 \mathrm{~dB}$ & $<0,05$ \\
\hline $\begin{array}{l}\text { Choun YH, y col } \\
2006\end{array}$ & $\begin{array}{l}39,4 \% \\
(13 \text { de } 33)\end{array}$ & $\begin{array}{l}6,1 \% \\
(2 \text { de } 33)\end{array}$ & $n / e^{3}$ & $9,1 \mathrm{~dB}$ & $2,4 \mathrm{~dB}$ & $\mathrm{n} / \mathrm{e}$ \\
\hline $\begin{array}{l}\text { Xenellis J, y col } \\
2006\end{array}$ & $\begin{array}{l}47,3 \% \\
(9 \text { de } 19)\end{array}$ & $\begin{array}{l}0 \% \\
(0 \text { de } 17)\end{array}$ & $<0,002$ & $14,8 \mathrm{~dB}$ & $-0,8 \mathrm{~dB}$ & $<0,0005$ \\
\hline
\end{tabular}

1: Se define como mejoría al menos $10 \mathrm{~dB}$ de PTP

2: $\mathrm{CIT}=$ Corticoides intratimpánicos

3: $n / e=$ No especificado 
Nuevamente la mayoría de los estudios que evalúan la efectividad de los corticoides sistémicos en esta enfermedad son de mala calidad metodológica, retrospectivos y sin grupo control. En general reportan mejorías que fluctúan entre 18,2 a $72 \%$ sobre el vértigo y 5 a $17 \%$ sobre la hipoacusia, variando según el tiempo de seguimiento y protocolo de administración utilizado.

Un estudio nacional de Riveros y cols, $2005^{30}$, muestra que sólo se obtienen beneficios en el vértigo (mejoría en el 79,4\% de los casos), no así en el tinnitus ni la hipoacusia en pacientes con EM en que han fallado otras terapias.

Destaca un estudio prospectivo, randomizado, doble ciego y cross-over (es decir, en que todos los participantes reciben en forma sucesiva el medicamento a prueba y el placebo o control, pero en orden distinto) que evalúa el uso de dexametasona con ácido hialurónico versus suero fisiológico con ácido hialurónico, todos intratimpánicos por tres días consecutivos, en 20 pacientes con EM probable 0 definitiva según los criterios de la Academia Americana de Otolaringología y Cirugía de Cabeza y Cuello. Este estudio no mostró beneficios de los corticoides intratimpánicos versus el grupo control en términos de resultados audiométricos, respuestas a la prueba calórica, y la presencia de tinnitus a las tres semanas de seguimiento ${ }^{31}$.

Otro estudio controlado randomizado doble ciego ${ }^{32}$ con un seguimiento de 2 años incluyó a 22 pacientes con EM que no estaban recibiendo otra forma de corticoides, y que fueron randomizados a dexametasona ( $4 \mathrm{mg} / \mathrm{mL}$ ) o suero fisiológico administrados por inyección intratimpánica durante 5 días consecutivos. A los 2 años de seguimiento $82 \%$ de los pacientes tratados con dexametasona intratimpánica mostraron control total del vértigo versus $47 \%$ en el grupo que recibió placebo. Además la dexametasona produjo una mejoría subjetiva en el tinnitus ( $48 \%$ versus $20 \%$ con placebo) y la hipoacusia (35\% versus $10 \%$ ). No se señala si las diferencias son estadísticamente significativas y hubo 4 personas del grupo control en que se tuvo que utilizar otras terapias para controlar el vértigo.

Esto sugeriría que los efectos de los corticoides intratimpánicos, si bien pueden no ser evidentes a las 3 semanas de seguimiento, puede que si lo sean a largo plazo (2 años). Sin embargo, esto no concuerda con otros estudios que muestran efectos a corto plazo y en el largo plazo este disminuye.

Vemos así que los resultados de los principales estudios no son concordantes, probablemente debido a la influencia de los factores mencionados al principio de este apartado. Aparentemente habría mayor efecto sobre el vértigo que sobre los otros síntomas de esta enfermedad.

\section{OTRAS PATOLOGÍAS}

Los corticoides intratimpánicos han sido utilizados en otras diversas patologías. Entre ellas se encuentra el tinnitus subjetivo de origen coclear, reportándose en algunos estudios sin grupo control una efectividad de $34 \%$ para hacer desaparecer el tinnitus y de $40 \%$ para disminuirlo. La efectividad era mayor en aquellos en que se inicia la terapia dentro de los primeros 3 meses de inicio de los síntomas ${ }^{33}$. Posteriormente estos hallazgos fueron desmentidos por un estudio randomizado, prospectivo y ciego ${ }^{34}$ que evaluó la efectividad de $0,5 \mathrm{~mL}$ de dexametasona $(4 \mathrm{mg} / \mathrm{mL})$ intratimpánico semanal por 4 veces versus placebo en 36 pacientes con tinnitus de origen predominantemente coclear. Veintidós por ciento de los pacientes que recibieron placebo y $33 \%$ de los pacientes que recibieron dexametasona mostraron mejoría de más de 2 puntos de escala visual análoga en forma inmediata (diferencia no estadísticamente significativa). Por otra parte, en el seguimiento a largo plazo (13 meses a 31 meses) los pacientes en que el tinnitus mejoró volvieron a su basal con el pasar del tiempo.

En animales se ha intentado probar su eficacia para reducir el daño histológico generado por el trauma acústico en ratas sin encontrar efecto significativo versus placebo $0^{35}$, pero sí para reducir la hipoacusia sensorioneural y los cambios morfológicos del oído interno inducidos por injuria acústica por el uso de fresado (similar al que se puede inducir durante algunas cirugías de oído) en cerdos de guinea ${ }^{36}$.

\section{CONCLUSIONES}

El uso de corticoides intratimpánicos en la práctica clínica ha suscitado gran controversia entre los 
otorrinolaringólogos, apareciendo tanto detractores como defensores de la técnica. Hemos visto que si bien permite alcanzar concentraciones mayores de corticoides en perilinfa y parece ser efectivo en el tratamiento de diversas patologías, los resultados obtenidos no son del todo concluyentes. Esto particularmente debido a la heterogeneidad en los preparados, protocolos de administración, etc.

Se requieren más estudios en que se usen protocolos y preparados que tengan la mayor efectividad esperada de acuerdo al análisis de los trabajos existentes, tanto clínicos como preclínicos. No cabe duda que al igual que con cada nuevo hallazgo terapéutico se hace necesario el estudio serio y responsable de la efectividad real de esta terapia, debiendo ser sometidos a prueba mediante estudios confiables de buena calidad metodológica para determinar en qué población específica son más beneficiosos.

\section{BIBLIOGRAFÍA}

1. Ітон A, Sakata E. Treatment of vestibular disorders. Acta Otolaryngol Suppl 1991; 481: 617-23.

2. Silverstein H, et al. Intratympanic Steroid Treatment of Inner Ear Disease and Tinnitus. Ear Nose Trota J 1996; 75: 468-71.

3. СHоI JY, ET AL. Dexamethasone increases fluid absorption via $\mathrm{Na}+\mathrm{H}+$ exchanger (NHE) 3 activation in normal human middle ear epithelial cells. Eur J Pharmacol 2006 Apr 24; 536 (1-2): 12-8.

4. Herman P, et al. Modulation of middle ear epithelial function by steroids: clinical relevance. Acta Otolaryngol 1997 Mar; 117 (2): 284-8.

5. Park SN, et AL. Effects of antibiotics and steroid on middle ear mucosa in rats with experimental acute otitis media. Acta Otolaryngol 2001 Oct; 121 (7): 808-12.

6. LIN J, ET AL. Dexamethasone inhibits mucous glycoprotein secretion via a phospholipase A2dependent mechanism in cultured chinchilla middle ear epithelial cells. Acta Otolaryngol 1997 May; 117 (3): 406-13.

7. McCormick DP, ET AL. Middle ear fluid histamine and leukotriene B4 in acute otitis media: effect of antihistamine or corticosteroid treatment. Int
J Pediatr Otorhinolaryngol 2003 Mar; 67 (3): 221-30

8. Shirwany NA, et al. Effect of transtympanic injection of steroids on cochlear blood flow, auditory sensitivity, and histology in the guinea pig. Am J Otol 1998 Mar; 19 (2): 230-5.

9. Sennaroglu L, et al. Effect of intratympanic steroid application on the development of experimental cholesteatoma. Laryngoscope 1998 Apr; 108 (4 Pt 1): 543-7.

10. FuKUSHIMA N, ET AL. Effects of intratympanic injection of steroids on changes in rat inner ear aquaporin expression. Acta Otolaryngol 2002 Sep; 122 (6): 600-6

11. LIU HJ, ET AL. Dexamethasone pharmacokinetics in Guinea pig inner ear perilymph. ORL $J$ Otorhinolaryngol Relat Spec 2006; 68 (2): 93-8

12. Gorcoolea MV. Clinical aspects of round window membrane permeability under normal and pathological conditions. Acta Otolaryngol 2001 Jun; 121 (4): 437-47.

13. Parnes LS, et al. Corticosteroid pharmacokinetics in the inner ear fluids: an animal study followed by clinical application. Laryngoscope 1999 Jul; 109 (7 Pt 2): 1-17.

14. Chen G, et AL. In vivo distribution and pharmacokinetics of dexamethasone sodium phosphate thermosensitive in situ gel following intratympanic injection. Sichuan Da Xue Xue Bao Yi Xue Ban 2006 May; 37 (3): 456-9

15. ChANDRASEKHAR SS, et AL. Dexamethasone pharmacokinetics in the inner ear: comparison of route of administration and use of facilitating agents. Otolaryngol Head Neck Surg 2000 Apr; 122 (4): 521-8.

16. NaCl Ozluoglu L, et al. Buffered papaverine facilitates passage of intratympanic dexamethasone to the inner ear. Acta Otolaryngol 2006 Dec; 126 (12): 1260-5.

17. Shulman A, et al. Intratympanic drug therapy with steroids for tinnitus control: a preliminary report. Int Tinnitus J 2000; 6 (1): 10-20.

18. BaRRS DM, ET AL. Intratympanic steroid injections for intractable Ménière's disease. Laryngoscope 2001 Dec; 111 (12): 2100-4.

19. Silverstein H, et al. Direct application of dexamethasone for the treatment of chronic eustachian tube dysfunction. Ear Nose Throat $J$ 2003 Jan; 82 (1): 28-32. 
20. Gouveris H, et AL. Intratympanic dexamethasone with hyaluronic acid in the treatment of idiopathic sudden sensorineural hearing loss after failure of intravenous steroid and vasoactive therapy. Eur Arch Otorhinolaryngol 2005 Feb; 262 (2): 131-4.

21. Ho GM, ET AL. Effectiveness of Intratympanic Dexamethasone Injection in Sudden-Deafness Patients as Salvage Treatment. Laryngoscope 2004; 114: 1184-9.

22. Choung YH, et AL. Intratympanic dexamethasone injection for refractory sudden sensorineural hearing loss. Laryngoscope 2006 May; 116 (5): 747-52.

23. Wei BPC, Mubiru S, O'Leary S. Steroids for idiopathic sudden sensorineural hearing loss. Cochrane Database of Systematic Reviews 2006, Issue 1. Art. No.: CD003998. DOI: 10.1002/14651858.CD003998. pub2.

24. KaKenAta S, et al. Comparison of Intratympanic and Intravenous Dexamethasone Treatment on Sudden Sensorineural Hearing Loss with Diabetes. Otol Neurotol 2006; 27: 604-8.

25. Choung YH, et AL. Intratympanic Dexamethasone Injection for Refractory Sudden Sensorineural Hearing Loss. Laryngoscope 2006; 116: 747-52.

26. John XenelLIS, MD, et AL. Intratympanic steroid treatment in idiopathic sudden sensorineural hearing loss: A control study. OtolaryngologyHead and Neck Surgery 2006; 134: 940-5.

27. KILIÇ R, E AL. Intratympanic Methylprednisolone for Sudden Sensorineural Hearing Loss. Otol Neurotol; 2007; 28: 312-6.

28. Hillman TM, ET AL. Intratympanic Steroids: Do They Acutely Improve Hearing in Cases of Cochlear Hydrops? Laryngoscope 2003; 113: 1903-7.
29. ArRiaga MA, et AL. Hearing Results of Intratympanic Steroid Treatment of Endolymphatic Hydrops. Laryngoscope 1998; Volume 108 (11, Part 1): 1682-5.

30. Riveros $H$ y COLS. Experience in the treatment of Ménière disease with trans-tympanic therapy. Rev. Otorrinolaringol. Cir. Cabeza Cuello 2005; 65: 187-92.

31. Silverstein H, et al. Dexamethasone inner ear perfusion for the treatment of Ménière's disease: a prospective, randomized, doubleblind, crossover trial. Am J Otol 1998 Mar; 19 (2): 196-201.

32. Garduno-Anaya MA, et al. Dexamethasone inner ear perfusion by intratympanic injection in unilateral Ménière's disease: a two-year prospective, placebo-controlled, double-blind, randomized trial. Otolaryngol Head Neck Surg 2005 Aug; 133 (2): 285-94.

33. Cesarani A, et al. Intratympanic dexamethasone treatment for control of subjective idiopathic tinnitus: our clinical experience. Int Tinnitus $J$ 2002; 8 (2): 111-4.

34. Araujo MF, et AL. Intratympanic dexamethasone injections as a treatment for severe, disabling tinnitus: does it work? Arch Otolaryngol Head Neck Surg 2005 Feb; 131 (2): 113-7.

35. YILDIRIM A. Effect of intratympanic dexamethasone on noise-induced temporary threshold shift. Laryngoscope 2005 Jul; 115 (7): 1219-22.

36. SCHNeider W, et al. The value of methylprednisolone in the treatment of an experimental sensorineural hearing loss following drill-induced ossicular chain injury: a randomized, blinded study in guinea-pigs. Acta Otolaryngol 1998 Jan; 118 (1): 52-5.

Dirección: Rubén González Echeverría

Manquehue Sur 1620, Departamento 102, Las Condes

Santiago, Chile

E mail: “mailto:rubensge@gmail.com” rubensge@gmail.com 